\title{
Polymer-surfactant assemblies in water. A SANS study
}

\author{
M.Y. LIN, S.K. SINHA and K. CHARI* \\ Exxon Research and Engineering Company, Annandale, New Jersey 08801, U.S.A. \\ * Eastman Kodak Company, Rochester, New York 14650, U.S.A.
}

\begin{abstract}
We study dilute solutions of poly(ethylene oxide) (PEO) and sodium dodecyl sulfate (SDS) mixture in water with small angle neutron scattering (SANS) experiments. The attachment of the surfactant molecules to the polymers in the form of micelles results in a repulsive interaction within the polymers, which in turn results in interesting behavior of the viscosity as a function of the SDS concentration. The effect of addition of salt to the solution is also investigated.
\end{abstract}

\section{INTRODUCTION}

When dissolved in water, many types of nonionic polymers will bind to the surfaces of ionic surfactant micelles, forming self-assembled complexes. The studies of such system have been quite extensive for the last twenty years. Shirahama and coworkers ${ }^{1}$ were the first to propose a "pearls on a necklace " model, suggesting that surfactant molecules form micelles when attached on the polymer strands. Subsequent NMR experiments on poly(vinylpyrrolidone) (PVP)-sodium dodecyl sulfate (SDS) and poly(ethylene oxide) (PEO)-SDS solutions have confirmed that SDS associated with PVP or PEO is indeed in the form of micelles. 2,3 Cabane and Duplessix have done small angle neutron scattering (SANS) experiments over a wide range of PEO and SDS concentrations. ${ }^{4-6}$ Specifically, they studied extensively with SANS the semidilute regime of PEO concentration, and suggested 3 distinct patterns of the PEO-SDS correlation. ${ }^{6}$

In this paper, we study the PEO-SDS system in the dilute regime of PEO concentration, below the overlapping concentration $\mathbf{y}^{*}$. Interesting questions remain as to how the polymer-surfactant correlation affects other physical properties. More specifically, as shown in Fig. 1, such solutions exhibit striking features in terms of their viscosity, which shows a peak and then a dip before finally increasing as a function of the SDS concentration. 7

With SANS experiments, by varying the contrast between the components and the solvent, we can probe the structure of either polymers or surfactants while the other is present. We found that individual polymers expand as the surfactant concentration is increased, up to a point where saturation of the absorption of the micelles to the polymers is reached. This point corresponds to the peak position of the viscosity in Fig. 1. After that, the polymers actually shrink, resulting in a decrease in the viscosity. The final rise in the viscosity is believed to be caused by a shape transition of the micelles from spherical to cylindrical, and is present even without polymers. 8

\section{EXPERIMENTAL}

Poly(ethylene oxide) (PEO) samples were obtained from Tosohaas Corporation. The dominant molecular weights used were $M_{w}=85,000$ with a polydispersity $M_{w} / M_{n}$ of 1.1 . The concentration of polymer in solutions used is $\mathbf{y}=0.01 \mathrm{~g} / \mathrm{cc}$ (We follow Cabane and Duplessix, using $\mathbf{x}$ and $\mathbf{y}$ as concentrations of surfactant and polymer, respectively), below the overlapping concentration of $y^{*}=0.06 \mathrm{~g} / \mathrm{cc}$. Deuterium labeled SDS (d-SDS) was obtained from MSD Isotopes. 
SANS experiments were performed at the National Institute of Standards and Technology's (NIST) Cold Neutron Research Facility, using the NIST/Exxon/University of Minnesota 30 Meter SANS instrument. The neutron wavelength was $\lambda=6 \AA$ with a spread of $\Delta \lambda / \lambda=0.14$. The range of the momentum transfer is $0.005<\mathrm{Q}<0.13 \AA^{-1}$. A solvent of $\mathrm{D}_{2} \mathrm{O}$ was used to almost match the neutron scattering length density of deuterium-labeled SDS (d-SDS) while the polymers were examined, so that d-SDS molecules were present but invisible to the neutrons.

\section{JII. RESULTS AND DISCUSSION}

In a SANS experiment, we measure the scattered neutron intensity $I(Q)$ as a function of the momentum transfer, $Q=4 \pi \sin (\theta / 2) / \lambda$, where $\theta$ is the scattering angle. Figure 2 shows a series of scattering curves of PEO taken at different d-SDS concentrations $x$ in a $\mathrm{D}_{2} \mathrm{O}$ solvent. Since the d-SDS molecules are invisible to the neutrons because of their match in neutron scattering length density with the solvent, the scattering curves reflect the spatial distribution of the polymers only. Two features are observed: First, the curves are peaked except the lowest and the highest SDS concentrations, and the peak moves outward as $\mathbf{x}$ increases; second, the overall intensity decreases as $\mathbf{x}$ increases.

We attribute the peak in the scattering to the correlations of the polymer segment groups associated with the attached micelles. The binding of a micelle to polymer results in a fraction of the polymer segments being wrapped around the micelle, and being carried around as the micelle makes movements. Since the micelles are charged and thus repulsive with each other, they try to form a short range ordered structure involving a characteristic nearest neighbor distance. This results in a similar structure for the polymer segment groups which the micelles carry around. The distance $\mathrm{d}$ between two adjacent polymer segment groups, and therefore that of two adjacent micelles, is reflected in the peak $\mathbf{Q}$ value $Q_{p}$, roughly as $d=2 \pi / Q_{p}$.

These interacting segment groups can belong to the same polymer, or come from different macromolecules, and which one is more important depends on the polymer concentration and the number density of polymers compared to the number density of the micelles. At $x=0.025 \mathrm{M}$, assuming an aggregation number of $n=100,5$ the ratio of the two densities is near unity, about one micelle per polymer. Therefore a segment group is the macromolecule itself, and the interaction is mostly intermolecular. The spacing of the polymers, $d=2 \pi / 0.027=233 \AA$, is close to the average distance of the polymers, obtained as $\left(\mathrm{yN}_{\mathrm{a}} / \mathrm{M}_{\mathrm{w}}\right)^{-1 / 3}=240 \AA$ where $\mathrm{N}_{\mathrm{a}}$ is the Avogadro constant.

As more d-SDS molecules are added to the solution, the number of micelles increases, while the number of polymers keeps the same. As a result, each micelle carries less amount of polymer segments, and the distance between them decreases. These two effects are reflected in the apparent drop in the scattering intensity and the move of the peak to a higher $Q$ value. The repulsive interactions become largely intramolecular. An important consequence of this intramolecular interaction is the expansion of the polymers, which in turn results in an increase of the viscosity as observed in Fig. 1.

The expansion of the macromolecules is also consistent with the decrease of the scattered intensity as $\mathbf{x}$ increases. We give a qualitative argument as follows. For a particular momentum transfer $Q$, we can roughly think of $\mathrm{I}(\mathrm{Q})$ as arising from the coherent scattering of all monomers within clusters of volume with radius $Q^{-1}$, and an incoherent superposition of the scattering of all such clusters. Thus if $m(Q)$ is the total monomer mass inside a cluster of radius $Q^{-1}$ and $M$ is the total mass in the scattering volume, the number of such clusters is $M / m(Q)$ and

$$
\mathrm{I}(\mathrm{Q}) \sim\left\{\mathrm{M} / \mathrm{m}(\mathrm{Q}) \times(\mathrm{m}(\mathrm{Q}))^{2}\right\} \sim \mathrm{m}(\mathrm{Q})
$$

(Such an argument would yield, for example, the well known scaling form $I(Q) \sim Q^{-d f}$ for a mass fractal of fractal dimension $d_{f}$ ). Thus for a given $Q, I(Q)$ is proportional to the average monomer density averaged over the volume of radius $Q^{-1}$. As the polymers expand, $I(Q)$ would decrease at large enough $Q$ $\left(\mathrm{Q} \gg \mathrm{Rg}^{-1}\right.$ ).

To check the above argument with the scattering data, we choose $Q=0.112 \AA^{-1}$, high enough to minimize the effect of repulsive interaction between segments, and focus on only the smallest length scales. We plot $1 /\left(\mathrm{Q}=0.112 \AA^{-1}\right)$ as a function of $x$ in Fig. 1 . We plot the inverse of the intensity instead of intensity itself is for comparison with the shape of the viscosity data in the same figure. The comparison should be only qualitative, that is, the function $1 / \mathrm{I}$ at high $\mathrm{Q}$ should follow the same trend of the polymer size, and hence that of the viscosity. However, the two curves are peaked at almost the same value of $\mathbf{x}$, and the similarity of the two curves is indeed striking. 
For the lowest d-SDS concentration $x=0.01 \mathrm{M}$, which does not exhibit a peak in the scattering, we also measured $I(Q)$ at a smaller $Q$ range. The fit of the data with a Debye (random coil) form shows that the average radius of gyration of the macromolecules actually decreased at $x=0.01 \mathrm{M}$ from their d-SDS free configuration. This suggests that the polymers first shrink before they expand as the d-SDS concentration is gradually increased, and is not totally unreasonable. At $x=0.01 \mathrm{M}$, the average number of micelles per polymer is much less than one, and the ionic interaction is largely absent. Since polymer segments prefer wrapping around the micelles, it may result in a smaller radius of gyration.

On the other hand, an interaction peak is also absent at the highest SDS concentration measured, $\mathbf{x}=0.2 \mathrm{M}$. It is also where the viscosity of the solution turns downward. We believe that the saturation of micelles on the polymers results in an excess surfactant concentration in solution, whose counter ions effectively screen the electrostatic interactions between the polymer-attached micelles.

Finally, the scattering intensity at high $Q$ follows as $I(Q) \sim Q^{-1 / u}$ where $u$ is the excluded volume exponent. 9,10 For the free polymer we obtain $1 / \mathrm{u}=1.7$, corresponding to a flexible polymer in a good solvent. For the saturated polymer at $x=0.15 \mathrm{M}$ we have $1 / \mathrm{u} \approx 1.55$ which is indicative of an expansion of the polymer coils. These values are also consistent with our viscosity measurements. ${ }^{7}$ Upon addition of salt (sodium chloride), however, the scattering exponent changes from $1 / \mathrm{u}=1.62$ at $0.005 \mathrm{M}$ salt concentration, to $1 / \mathrm{u}=1.73$ at $0.1 \mathrm{M}$ salt concentration. The extra counter ions provided by salt screens the double layer electrostatic interaction of the attached micelles, thus decreasing the degree of the expansion of the polymers.
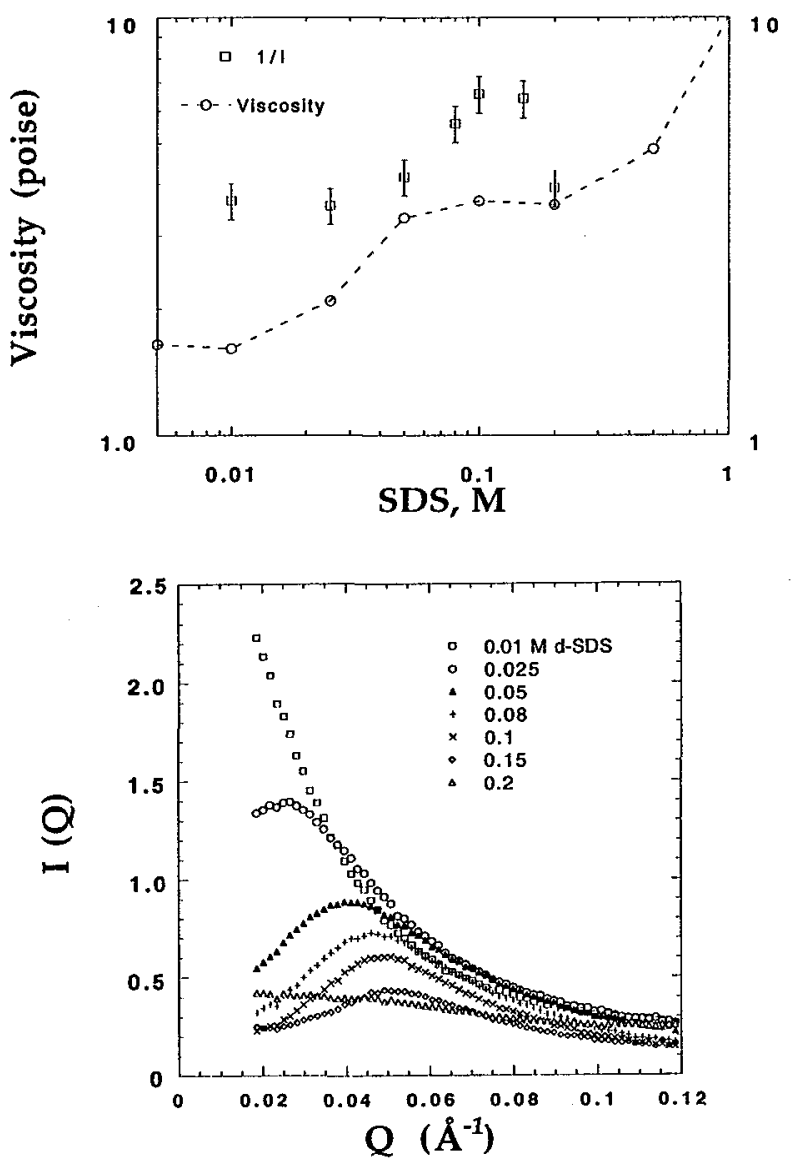

Figure 1. The viscosity of the PEOSDS solution as a function of SDS concentration (Circles connected by dashed line, left scale). The PEO is of molecular weight of 160,000 and concentration $y=0.005 \mathrm{~g} / \mathrm{cc}$. Also plotted is the inverse of the scattering intensity at $\mathrm{Q}=0.112 \AA^{-1}$ (squares, right scale) to show the trend of polymer size as a function of $\mathbf{y}$ for comparison (see text).

Figure 2. Neutron scattering intensity $I(Q)$ as a function of the scattering vector $Q$ for $P E O$ of molecular weight of 85,000 at various levels of d-SDS. 


\section{REFERENCES}

[1] SHIRAHAMA K., TSUJI, K., and TAKAGI, T.,J. Biochem. 75, (1974) 309.

[2] CANABE B., j. Phys. Chem. 81 (1977) 1639.

[3] CHARI K., J. Colloid Interface Sci. 151 (1992) 294.

[4] CABANE B. and DUPLESSIX R., J. Phys. (Paris) 43 (1982) 1529.

[5] CABANE B. and DUPLESSIX R.,Colloids Surf. 13 (1985) 19.

[6] CABANE B. and DUPLESSIX R., J. Phys. (paris) 48 (1987) 651.

[7] CHARI K., ANTALEK B., LIN M.Y., and SINHA S.K., to be published.

[8] PRASAD CH.D. and SINGH H.N., Colloids Surf. 59 (1991) 27.

[9] DEGENNES P.G., Scaling Concepts in Polymer Physics (Cornell University, Ithaca, 1979).

[10] COTTON J.P., DECKER D., FARNOUX B., JANNINK G., OBER R., and PICOT C., Phys. Rev. Lett. 32 (1974) 1170. 